\title{
Stability and Gait Transition of the Five-Link Biped on Stochastically Rough Terrain Using a Discrete Set of Sliding Mode Controllers
}

\author{
Cenk Oguz Saglam ${ }^{1}$ and Katie Byl ${ }^{1}$
}

\begin{abstract}
The five-link biped is a simple, planar model of human-like walking in which scuffing can be avoided. In this paper, we focus on controller design and stability analysis for the important case of non-steady walking, toward such goals as avoiding obstacles on terrain or meeting specific requirements in speed or energetics. To achieve such tasks as new sensor information about upcoming terrain becomes available, control must be adjusted on-the-fly, preferably using a continuous family of controllers. Here, we present an illustrative case using only two, discrete sets of controllers and investigate the effect of switching between them on a stochastically rough terrain. Of note, we find that the tenth-order system dynamics of unsteady walking can be accurately represented as a Markov process, using only a sparse, quasi-2D mesh of discrete states. This transition matrix approach is then used to determine bounded limits on terrain noise for which guarantees of stability (i.e., never falling) may be given for a particular controller and for arbitrary switching between the controllers, as well as to estimate fall rates for cases where these bounds are exceeded. Our results also allow us to quantify the increase in stability gained by a simple policy of switching based on a noisy, singlestep lookahead on terrain. This illustrative example, using two controllers that behave differently and allow for arbitrary switching, provides a framework for future work where tasks or requirements for biped walking are clearly defined and can only be achieved by a wider set of, or ideally a continuous range of, controllers.
\end{abstract}

\section{INTRODUCTION}

Locomotion is an undeniable necessity for many robot application. Bipedal robot walking is an anthropomorphic approach, providing an intuitive means for negotiating intermittent or otherwise rough terrain, where wheels would be ineffective. For development of such robots, use of underactuated, dynamic walking gaits provides a practical means of operating in environments with extreme perturbations [1], [2] and may be essential in eventually reducing energetic cost for such robots [3], [4]. In recent years, several control approaches have been proposed to address the ongoing challenge of non-steady, underactuated biped walking on rough terrain [5], [6], [7], [8], [9], [10], [11], [2]. The current paper addresses the challenges of addressing increasingly high-dimensional legged systems and of quantifying tradeoffs between stability and other desired performance metrics.

In this work, we address the problems of using noisy information about upcoming rough terrain both to select

This work was supported by the Institute for Collaborative Biotechnologies through grant W911NF-09-0001 from the U.S. Army Research Office. The content of the information does not necessarily reflect the position or the policy of the Government, and no official endorsement should be inferred.

${ }^{1}$ C.O. Saglam and K. Byl are with the Electrical and Computer Engineering Department, University of California, Santa Barbara, CA 93106 USA saglam at ece.ucsb.edu, katiebyl at ece.ucsb.edu foothold width to avoid obstacles and bad terrain and to modify the gait to accommodate variations in terrain height. Important contributions are the use of stochastic modeling tools both to define bounds on the noise about upcoming terrain for which control will remain stable and to quantify the rate of failure when these bounds are exceeded. In particular, we report on the use of low-dimensional (2D) meshing in representing much higher-order (10D) system dynamics, extending methods presented in [12] and [13].

The rest of the paper is organized as follows. We first present briefly the robot used in simulations and then offer a control scheme that allows us to easily map the tasks to controller output. We then integrate sliding mode controllers (SMC) to this scheme. We use the states just before the impacts to create a discrete Poincaré map. After verifying the stability of individual controllers through simulation, we test the robot with randomly switching controllers and use Poincaré map to interpret the results. Then we calculate numeric solutions for the state to state transitions for each controller for a wide range of slopes to capture whole dynamics of the robot and create a transition matrix for a set of mesh points along the surface of Poincaré states. This approach allow us to determine bounded limits on terrain noise for which the walker can be guaranteed to never fall, and it also provides a helpful approach for determining which controller to use, given a short lookahead of information about upcoming terrain slope. These results are tested and verified using simulations on grounds modeled by normal distributions of different means and deviations. Finally, we discuss two specific applications that motivate our approach: (1) the ability to adjust footstep length on-the-fly, to avoid upcoming terrain obstacles, and (2) switching controllers to adapt to upcoming changes in slope.

\section{MODEL}

\section{A. Definitions}

The five-link model used in this paper is depicted in Fig. 1. We use relative angles $q:=\left[\begin{array}{lllll}q_{1} & q_{2} & q_{3} & q_{4} & q_{5}\end{array}\right]^{T}$ and restrict our attention to planar motion. This is a slightly edited version of the RABBIT biped [14], and model parameters used are listed in Table I.

As illustrated in Figure 1 and Table I, we refer to the upper leg links associated with $q_{1}$ and $q_{2}$ as femurs. The torso is the uppermost element, and the tips are the remaining two. We assume point masses between the joints but not at the hip. The walking motion is a sequence of steps which have hybrid dynamics, described by a continuous swing phase and a discontinuous impact event. 

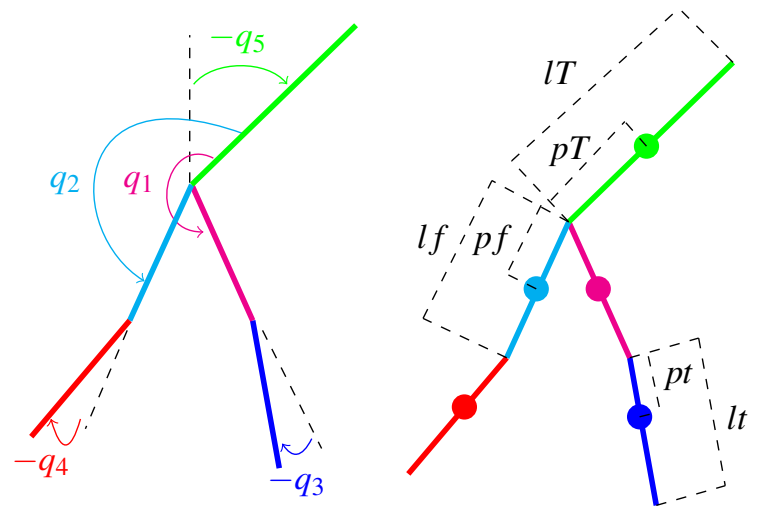

Fig. 1. Illustration of the five-link robot with identical legs

TABLE I

Model Parameters for the Five-Link Robot

\begin{tabular}{|c|c|c|}
\hline Description & Parameter Label & Value \\
\hline Torso Mass & $m_{T}$ & $12 \mathrm{~kg}$ \\
\hline Femur Mass & $m_{f}$ & $6.8 \mathrm{~kg}$ \\
\hline Tip Mass & $m_{t}$ & $3.2 \mathrm{~kg}$ \\
\hline Torso Inertia & $I_{T}$ & $1.33 \mathrm{~kg} \mathrm{~m}$ \\
\hline Femur Inertia & $I_{f}$ & $0.47 \mathrm{~kg} \mathrm{~m}^{2}$ \\
\hline Tip Inertia & $I_{t}$ & $0.20 \mathrm{~kg} \mathrm{~m}$ \\
\hline Torso Length & $l_{T}$ & $0.63 \mathrm{~m}$ \\
\hline Femur Length & $l_{f}$ & $0.4 \mathrm{~m}$ \\
\hline Tip Length & $l_{t}$ & $0.4 \mathrm{~m}$ \\
\hline Torso Mass Center & $p_{T}$ & $0.24 \mathrm{~m}$ \\
\hline Femur Mass Center & $p_{f}$ & $0.11 \mathrm{~m}$ \\
\hline Tip Mass Center & $p_{t}$ & $0.24 \mathrm{~m}$ \\
\hline Gravitational Acceleration & $g_{0}$ & $9.81 \mathrm{~m} / \mathrm{s}^{2}$ \\
\hline Coefficient of friction & $\mu_{s}$ & 0.7 \\
\hline
\end{tabular}

\section{B. Swing Phase}

We define the swing leg to consists of the femur and tip associated with $q_{2}$ and $q_{4}$, respectively. The other femur and tip form the stance leg. At each impact event, the previous swing leg becomes the new stance leg and vice versa, and so the two legs are relabeled. Here, we concentrate on the continuous part of one step which is between two impacts. As the name dictates, swing phase refers to swing leg taking off from the ground, passing the stance leg and landing on a further point on the ground. We use the following canonical form to model the continuous dynamics:

$$
D(q) \ddot{q}+C(q, \dot{q}) \dot{q}+G(q)=B u
$$

where matrices $D, C, G$ and $B$ are derived using the Lagrangian approach. We can define $x=\left[\begin{array}{ll}q^{T} & \dot{q}^{T}\end{array}\right]^{T}$ to express the dynamics equivalently as:

$$
\dot{x}=\left[\begin{array}{c}
\dot{q} \\
D^{-1}(-C \dot{q}-G+B u)
\end{array}\right]=: f(x)+g(x) u .
$$

An important point is that $q$ consists of the five angles depicted in Fig. 1 whereas $u$ has only four elements. The system has a degree of underactuation of one due to the fact that we have a passive joint at the stance tip. Also note that we have five degrees of freedom instead of seven because the position of the stance tip end, the pivot point in contact with the ground, is constant during the motion. As mentioned, the swing phase starts with an impact and ends with another. Although the controllers are effective during the swing phase, the effects of the impact event will be the main focus in our controller design.

\section{Impact Event}

Although the dynamics of the impact event itself may contribute to step-to-step variability in real-world legged robotics [15], [16], in this paper, we use the classic impact model formulated in [17] and used in [14]. Essentially, this model assumes instantaneous, inelastic collisions between the swing leg tip and the ground, with the instantaneous changes in velocities to reflect the effects of impulsive forces exerted on the robot. We denote the states just before and after the impact as $x^{-}$and $x^{+}$respectively. The impact model is a mapping from $x^{-}$to $x^{+}$, i.e., $x^{+}=\Delta\left(x^{-}\right)$. The changes in the velocities are calculated using conservation of energy and the principle of virtual work. This model is discussed in great detail in [17]. The angles undergo a comparatively simple transformation at impact: although the robot's position and orientation do not actually change according to the impact model, we relabel the legs so that the swing leg will always be the one parametrized by the $q_{2}$ and $q_{4}$. That is:

$$
\left[\begin{array}{lllll}
q_{1}^{+} & q_{2}^{+} & q_{3}^{+} & q_{4}^{+} & q_{5}^{+}
\end{array}\right]=\left[\begin{array}{lllll}
q_{2}^{-} & q_{1}^{-} & q_{4}^{-} & q_{3}^{-} & q_{5}^{-}
\end{array}\right]
$$

As a result we will always have the leg associated with $q_{2}$ and $q_{4}$ as the swinging one.

\section{Hybrid Model}

The two parts of the hybrid model described below are linked as shown in Fig. 2. After an impact, the robot swings its leg until another impact occurs, i.e., until the swing tip touches the ground. A step consists of an impact event and a swing phase. In our modeling, we assume the impact event occurs first, but the order in the definition of a step is an arbitrary choice, so long as one remains consistent after deciding. The importance of the impact can be better

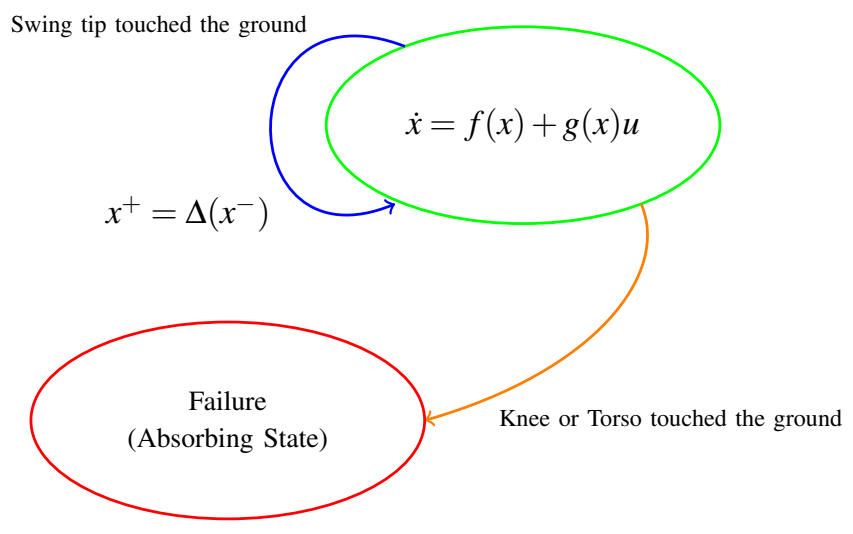

Fig. 2. Hybrid model of a step and failure state

understood when noted as reinitialization for the swing 
phase. If the reinitialized robot can successfully complete the next step for all allowable (bounded) stochastic terrain events, we call $x^{-}$a safe state. Thus a controller has two main objectives: Choosing an $x^{-}$and converging to it. The following section will explain what makes a state safe.

\section{CONTROL}

\section{A. Limitations and Goal}

As explained in the previous section, the robot is underactuated by one degree during the swing phase. As a result, the five angles, $q$, should be combined to choose a set of four variables, $q_{c}$, to be controlled. The choice of possible variables is not unique and may include coordinates on the body, such as the position of the center of mass, toward driving the system in stable, forward motion. In the present work, however, we simply chose $q_{c}:=\left[\begin{array}{llll}q_{2} & q_{3} & q_{4} & q_{5}\end{array}\right]^{T}$ to be the parameters to be controlled. This idea originates from our observations on experiments using the three-link walker where we decided to control the swing leg and torso angles but not the stance leg directly. We observed that the velocity of the stance leg was almost always negative (except for poor initial conditions), which meant a monotonic decrease in the stance leg angle during a successful step. This is also the case here as shown in Fig. 3, where a typical step is given. $\dot{q}_{1}$ is mostly negative and also nearly constant for a considerable time. With this choice of $q_{c}$, invertibility of the decoupling matrix is then exploited in determining the four control torques, $u$, as described in [14] and reviewed briefly in Section III-B. Note that given enough time, $q_{1}$

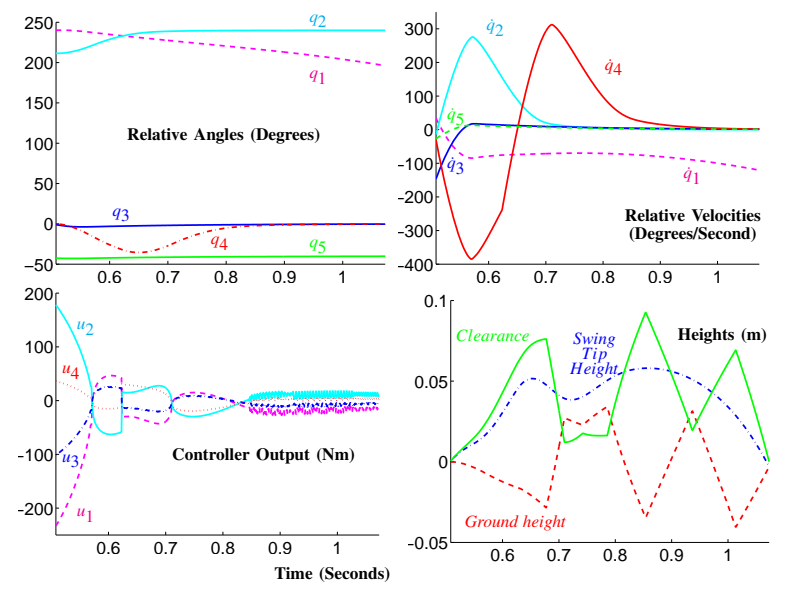

Fig. 3. A typical step

achieves the desired reference we have in mind, determined by the other references imposed on $q_{c}$, so long as the slope of the terrain ahead is accurately known. That is, $q_{c}$ and the terrain geometry at the moment of impact uniquely determine $q_{1}$. Concentrating on the remaining four angles makes the controller design method very easy and straightforward. A negative but small-magnitude $\dot{q}_{1}$ gives us a reasonable time to move robot to the state we want in the ten dimensional space. The main goal of the controller is to make $q_{c}=q_{c}^{r e f}$ before the impact occurs. Reaching our references rapidly will also put $\dot{q}_{c}$ close to zero, and this is in fact one of the most important criteria determining the success of our step. This will be explained in more detail in the context of simulation results. However, we note there is a direct trade-off between achieving fast convergence and the limiting the magnitude of the required controller outputs (i.e., motor torque limits).

We now further investigate Fig. 3 to explain controller goals for each state variable intuitively. We prefer $q_{2}$ to move fast, particularly on rough terrain, where the impact event may occur earlier than anticipated. Compared with the other angles, it can be seen that $q_{4}^{\text {ref }}$ (lower swing leg tip) changes quite dramatically throughout a step: $q_{4}$ is first sent to a negative value and then forced back to zero before the impact occurs, to insure ground clearance of the swing leg. The negative reference and the time when we start going back can be adjusted to have a larger clearance for the swing leg or smaller controller effort. Note that we send $q_{4}$ to zero because it will be relabeled as $q_{3}$ in the next step, and we want $q_{3}$ to be always zero for again ground clearance. The torso angle, $q_{5}$, can be used to bias forward motion and serves as a parameter to set the duration of a step. In [13], a bent torso in a three-link walker significantly enhanced robustness to terrain height variations during blind walking, compared with upright walking. Use of the torso in this work is similarly inspired but somewhat more complicated in practice. On one hand, the center of mass depends highly on torso angle, i.e., leaning forward speeds up the robot by moving the center of mass forward: if the torso does not lean forward enough, we may fall backward rather than completing a forward step. However, a high forward speed may also prevent the robot from reaching its reference before impact, thus also causing instability, as we will see. Finally, note the ground clearance is defined as a non-negative swing tip height above ground, and that impact occurs when it becomes zero. We have verified the intuitive ideas described above in numerous simulations, using different ground profiles, model parameters and initial conditions.

\section{B. Control Action during the Swing Phase}

We select the input to the system to be in the form of

$$
\begin{gathered}
u=\left(S D^{-1} B\right)^{-1}\left(v+S D^{-1}(C \dot{q}+G)\right) \\
\text { where } S=\left[\begin{array}{lllll}
0 & 1 & 0 & 0 & 0 \\
0 & 0 & 1 & 0 & 0 \\
0 & 0 & 0 & 1 & 0 \\
0 & 0 & 0 & 0 & 1
\end{array}\right]
\end{gathered}
$$

and D, B, C, G are derived by the Lagrangian approach. Substituting this $u$ into (1) and noting $q_{c}=S q$ by their definitions, we obtain a very simple equation for the second derivatives of the angles to be controlled:

$$
\ddot{q}_{c}=v
$$

Note that $\ddot{q}_{1}$ is not included in this equation and it is not directly controlled as explained in the previous section. Since $q_{1}$ plays an important role in the step time, fast convergence will play a critical role in many aspects including the 
stability. After getting the simple structure of (6), what is left to do is to select $v$ appropriately, to ensure fast convergence to given references with acceptable torque inputs. Adopting the control outlined in [18], to have finite time convergence we first define the error to be the difference between the given reference and actual output.

$$
e_{i}=q_{i}-q_{i}^{r e f}, \quad i=\{2,3,4,5\}
$$

Thus we have 4 decoupled error dynamics. We then define generalized error to be

$$
\sigma_{i}=\dot{e}_{i}+e_{i} / \tau_{i}, \quad i=\{2,3,4,5\}
$$

where $\tau_{i}$ s are the time constants to be selected. Finally, we select $v^{T}=\left[\begin{array}{llll}v_{1} & v_{2} & v_{3} & v_{4}\end{array}\right]$ to be

$$
v_{i}=-k_{i}\left|\sigma_{i}\right|^{2 \alpha_{i}-1} \operatorname{sign}\left(\sigma_{i}\right)
$$

where $i=\{2,3,4,5\}, k_{i}>0$ and $0.5 \leq \alpha_{i}<1$. The control parameters used are given in Table I.

TABLE II

CONTROLler Parameters

\begin{tabular}{|c|c|c|c|}
\hline$i$ & $\tau$ & $\mathrm{k}$ & $\alpha$ \\
\hline 1 & $1 / 15$ & 50 & 0.75 \\
\hline 2 & $1 / 5$ & 50 & 0.75 \\
\hline 3 & $1 / 13$ & 50 & 0.75 \\
\hline 4 & $1 / 5$ & 25 & 0.75 \\
\hline
\end{tabular}

Two discrete sets of references used, as given in Table III. We refer to these as Controller 1 and Controller 2, using the first and second reference set, respectively. We could of course optimize controller parameters for each reference set; however, it is more illustrative for our purposes here to keep them as shown, toward quantitative comparison of the two. Note that both controllers were able to work quite successfully on moderately rugged stochastic terrain.

TABLE III

REFERENCE SETS

\begin{tabular}{|c|c|c|c|c|c|}
\hline Set & $q_{2}^{\text {ref }}$ & $q_{3}^{\text {ref }}$ & $q_{4}^{\text {ref } 1}$ & $q_{4}^{\text {ref } 2}$ & $q_{5}^{\text {ref }}$ \\
\hline 1 & 267.5 & 0 & -45 & 0 & -60 \\
\hline 2 & 240 & 0 & -45 & 0 & -40 \\
\hline
\end{tabular}

Note in Table III that we impose two different reference values for the lower swing leg angle, $q_{4}$, during a step, i.e.:

$$
q_{4}^{r e f}= \begin{cases}q_{4}^{r e f 1}, & \text { condition } \\ q_{4}^{r e f 2}, & \text { otherwise }\end{cases}
$$

where we selected condition, above, to be " $q_{2}<q_{1}$ ". Both the first reference, $q_{4}^{r e f 1}$, and the condition can be adjusted to enable either a larger ground clearance for the swing leg or a smaller controller effort. However, the primary goal in our work is to avoid early impacts, for stability on geometrically rough terrain. These parameters can also be adjusted adaptively during a step, in a manner analogous to a human responding during blind walking.

\section{SIMULATION}

\section{A. Stability of the Controllers}

For our initial tests of the stability of the two controllers, we first define the ground profile, $g(x)$, to be:

$g(x):=10^{-3}(3 \sin (\sqrt{80} x)+1.5 \sin (\sqrt{270} x)+1.5 \sin (\sqrt{531} x))$

Note that $g(x)$ is not periodic and has a small (sub-cm) amplitude. After verifying both controllers were able to take thousands of steps when used individually on the ground function $g(x)$, we ran simulations again, selecting a controller randomly at each step and recording $x^{-}$values, to plot a Poincaré map. The first thousand points are shown in Fig. 4. We select $q_{1}$ and $\dot{q}_{1}$ as the independent axes, observing that all other variables could be quite accurately determined as a function of these two values and the controller type used. In other words, the Poincarè states for each controller lie on a $2 \mathrm{D}$ manifold within the $10 \mathrm{D}$ state space. $q_{3}, q_{4}$ and $\dot{q}_{c}$ are plotted to show that they are almost zero. They will correspondingly not be examined in detail after this.
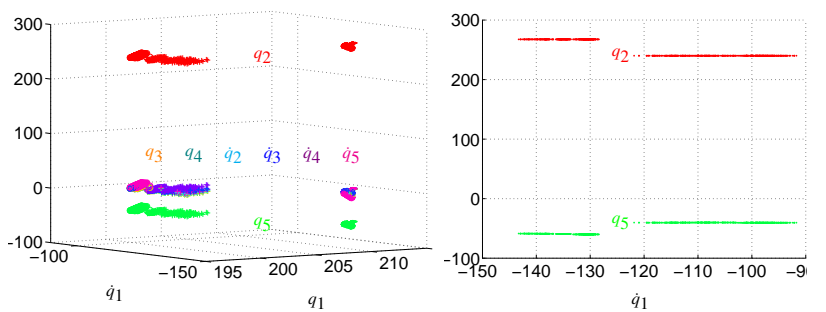

Fig. 4. Poincaré Sections. Angles are in degrees and velocities are in degrees/second. Indistinguishable values $q_{3}, q_{4}, \dot{q}_{2}, \dot{q}_{3}, \dot{q}_{4}$ and $\dot{q}_{5}$ are given to show they are effectively zero for this ground and controller set

Since the amplitude of the selected ground is small, we do not encounter early impacts and have enough time to converge to the references. That is the reason why $\dot{q}_{c}, q_{3}$ and $q_{4}$ are very close to zero and why we observe flat surfaces for $q_{2}$ and $q_{5}$. The $2 \mathrm{D}$ structure of the Poincarè states allows us to mesh the step-to-step stochastic dynamics of this system using an irregular and relatively sparse mesh of observed, discrete states. This in turn allows us to test the limits of the controllers by completely forming the transition matrix of the "steps that can be taken" given any particular stochastic description of terrain, as we do next.

\section{B. Transition Matrix of Step-to-Step Dynamics}

To build a transition matrix of the Markov process for walking on stochastic terrain, we begin with the data for 100 observed $x^{-}$states, from the simulation described above, along with a single, absorbing failure state. All failed steps transition to this failure state, which in turn can transition only back to itself. For each controller, we simulate a single step from each of these points for twenty one slopes ranging from $-10^{\circ}$ to $+10^{\circ}$. If a step is successfully taken, we decide whether the resulting pre-impact state is close enough to one of the existing states or if we need to create a new mesh point 
to represent the transition. To determine this, we define $P$ to be the set of existing mesh points and calculate:

$$
d_{\text {closest }}(x):=\min _{p \in P}\{d(x, p)\}
$$

where

$$
d(x, p):=\sum_{i=1}^{10}\left(\frac{x_{i}-p_{i}}{R_{i}}\right)^{2}
$$

where radius $R$ is chosen to be as in Table IV and a point is created when $d(x)>2.89$. Note the scaling factor of $10^{3}$ in Table IV, and that units here are given in radians and $\mathrm{rad} / \mathrm{sec}$, as appropriate. We repeat this simulation procedure for the newly added points until number of the points stops increasing, i.e., until the mesh states form a closed set. In this work, we err on the side of a more densely-packed mesh than is likely necessary: the resulting set, $P$, has 18,934 points.

TABLE IV

RADII FOR THE DISTANCE CHECK

\begin{tabular}{|c|c|c|c|c|c|c|c|c|c|c|}
\hline$i$ & 1 & 2 & 3 & 4 & 5 & 6 & 7 & 8 & 9 & 10 \\
\hline $10^{3} R_{i}$ & 3.5 & 12.6 & 2.6 & 4.7 & 1.4 & 36 & 2.4 & 15.4 & 66.6 & 2.4 \\
\hline
\end{tabular}

In Fig. 4 , we chose $q_{1}$ and $\dot{q}_{1}$ for our axes because ground variability was minimal, giving the swing leg tip $\left(q_{4}\right)$ ample time to arrive near its reference value of zero. While the same choice can still be adopted here as well, we now use $\dot{q}_{4}$ instead of $\dot{q}_{1}$ as it varies more as terrain slope increases in variability, due to the way we control the swing leg, $q_{4}$. In addition, $q_{1}$ is more strongly correlated with $\dot{q}_{1}$ than with $\dot{q}_{4}$, making the later a more practical independent coordinate for illustrating our results. We present the angles in Fig. 5.
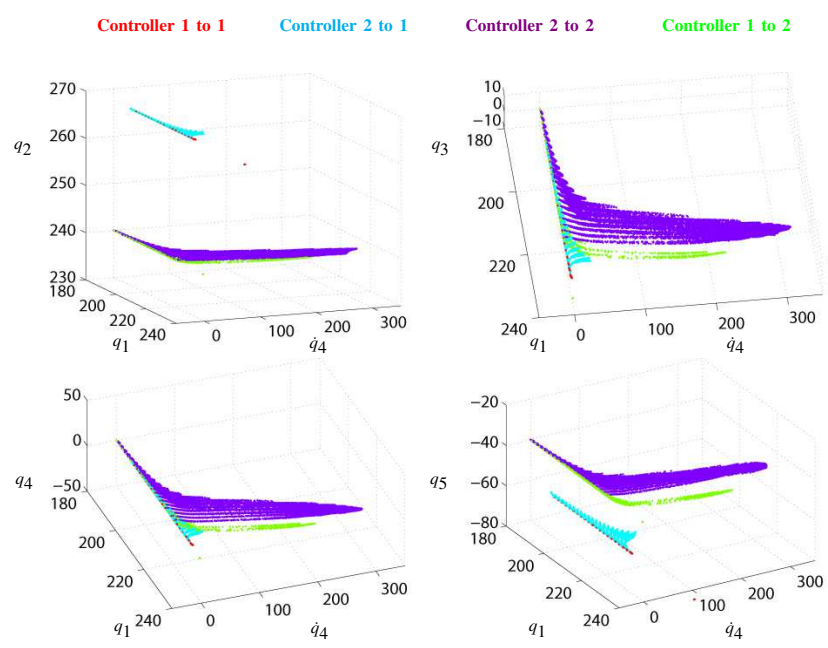

Fig. 5. Poincaré sections showing controller effect on next pre-impact state. Angles are in degrees and velocities are in $\mathrm{deg} / \mathrm{sec}$.

In Fig. 5, four colored regions are illustrated, each representing a switching sequence of the controller used on the previous and current steps for each pre-impact state plotted. Looking at the $\mathrm{z}$ scale, note that state $q_{3}$ (stance leg tip) is always relatively small (as might be expected).
The negative values of $q_{4}$ indicate premature impacts, due to high, upcoming ground slopes. Note that even with switching between controllers, $q_{2}$ and $q_{5}$ form distinguishable, flat surfaces. If this were not true, the mesh would rapidly fill a larger volume of state space, and our methods would likely be impractical for the case of switching among larger sets distinct controllers. Instead, we observe a rapid convergence of each controller to its own 2D manifold, meaning our toy example can likely be extended to the case of many distinct controllers, each tuned for a particular step width and/or to remain stable for a particular upcoming terrain slope.

Fig. 6 shows pre-impact velocities for the same mesh points, $P$. We note the lines in the upper-left figure result

$$
\text { Controller } 1 \text { to } 1 \text { Controller } 2 \text { to } 1 \quad \text { Controller } 2 \text { to } 2 \quad \text { Controller } 1 \text { to } 2
$$
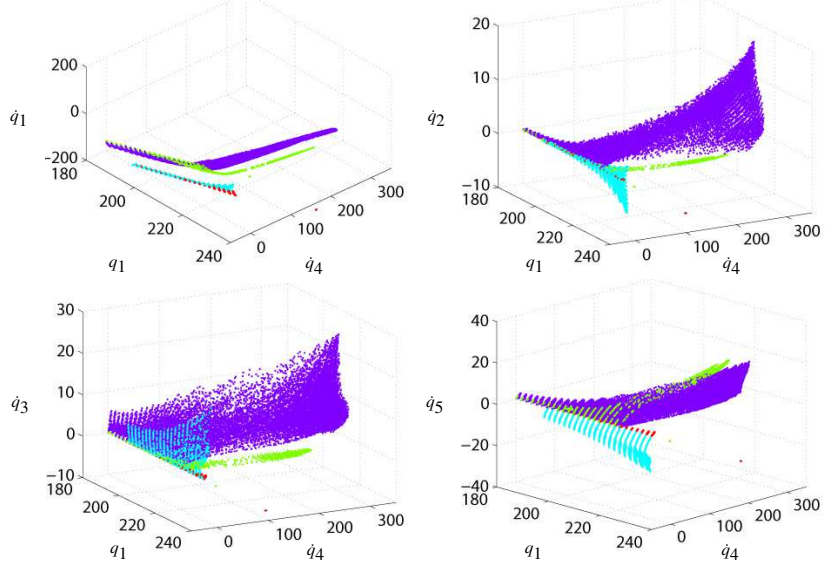

Fig. 6. Poincaré sections showing controller effect on velocities. Angles are in degrees and velocities are in degrees/second.

from the discrete nature of the slope range that we used. We can state $\dot{q}_{2}, \dot{q}_{3}$ and $\dot{q}_{5}$ stay relatively small compared to $\dot{q}_{1}$. The variance in stance leg velocity, $\dot{q}_{1}$, is simply a result of impacts occuring over a range of different terrain slopes.

We are now ready to present a set of very explanatory graphs which were mentioned in previous sections. For a given definition of bounded terrain noise, we classify a state $x^{-}$as "safe" if we can guarantee achieving a step with the corresponding $x^{+}$as initial condition to the swing phase. Similarly we say a point $x^{-}$is "unsafe" if such guarantees can not be made. Note that in our particular example, most of the unsafe states are in fact guaranteed to fail within the next step or two. Figs. 7 and 8 show regions classified in light of this understanding. Both controllers have their own, individual safe and failure regions. In Figs. 7 and 8, we are illustrating as safe only those initial conditions that are guaranteed to never fail given any arbitrary switching between controllers on future steps. We illustrate these regions for the largest bounded limits on upcoming terrain slope at each step for which any guarantee of stability (i.e., never falling) can be made: specifically, terrain slope is bounded to be between -5 and +3 degrees at each step here. By comparison, the bounds required to guarantee stability when using Controller 1 alone are -7 to +4 degrees; for Controller 
Unsafe Region
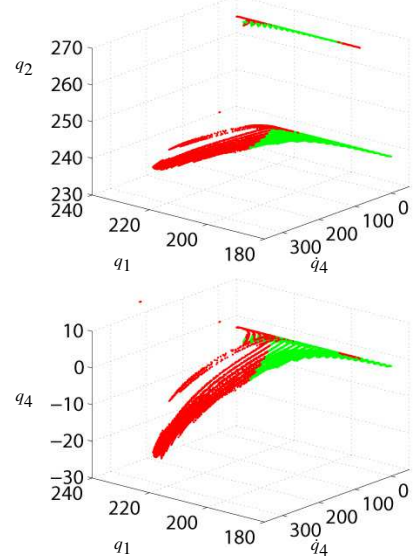

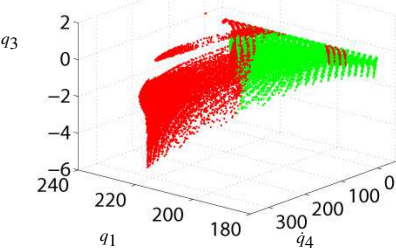

Safe Region

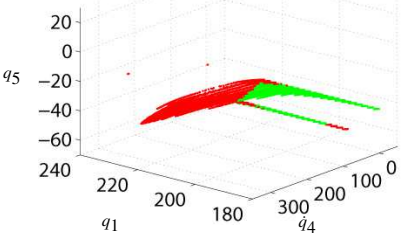

Fig. 7. Poincaré sections showing safe and failure regions for angles. Angles are in degrees and velocities are in degrees/second.

2 , they are -10 to +3 degrees. We note that large swing tip velocity, $\dot{q}_{4}$, is indicative of eminent failure in the following step. This is expected since $\dot{q}_{4}$ is large while $q_{4}$ is still moving between two references, meaning impact occurred before $q_{4}$ settles. For improved robustness on stochastic terrain, we can improve our controllers, which is a subject for future work. These figures motivate us to design a large set of discontinuous or (potentially) continuous controllers to transition within safe regions to achieve different objectives such as obstacle avoidance, perturbation recovery, and energy modulation as terrain height varies.
Unsafe Region
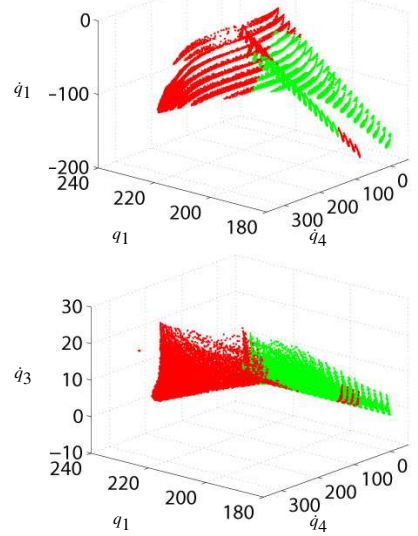
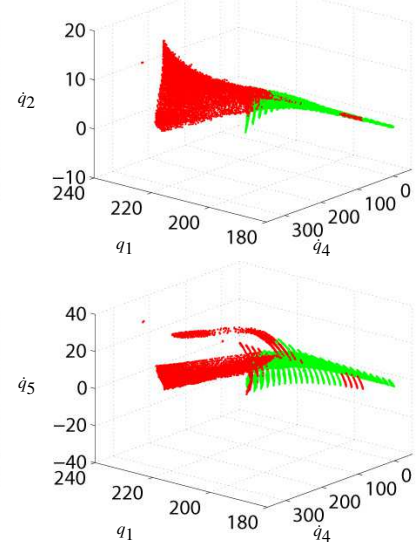

Fig. 8. Poincaré sections showing safe and failure regions for velocities. Angles are in degrees and velocities are in $\mathrm{deg} / \mathrm{sec}$.

\section{Theorotical Estimation of Number of Steps Before Failure}

While forming the set of mesh points, we record a map matrix $M$, which has the following structure: For the selected controller, given slope and starting point $p^{\text {start }}$, we estimate the new point $p^{e s t}$ as:

$$
p^{e s t}= \begin{cases}M\left(p^{a p p}, \text { slope } e^{a p p}\right), & \text { Controller } 1 \text { used } \\ M\left(p^{a p p}, 21+\text { slope }^{a p p}\right), & \text { Controller } 2 \text { used }\end{cases}
$$

where $M(i, j)$ is the element on the $i$ th row and $j$ th column, $p^{e s t}$ is the estimated new point, $p^{a p p}$ is the $p \in P$ which minimizes the distance $d(x, p)$ defined in (13) and slope $e^{a p p}=$ $11+\min (10, \max (-10, \operatorname{round}($ slope $)))$ is the approximated slope index.

Matrix $M$ allows the state evolution to be interpreted as a Markov Chain. We can then create a state transition matrix, $T$, once some stochastic description of the probability of each next terrain slope is given. $T$ will be $(n+1) \times(n+1)$ where $n=18934$ is the number of points we obtained before and +1 is due to the addition of an absorbing state, i.e. failure, in the mesh. As explained in detail in [12], expected mean of the steps before failure $N$ can be approximated by

$$
N \approx 1 /\left(1-\lambda_{2}\right)
$$

where $\lambda_{2}$ is the second largest eigenvalue of the transition matrix $T$. Results for different terrain slope means, standard deviations, and controllers are plotted in Fig. 9. We observe Controller 1 is more stable for slopes with mean around zero. We also note that with Controller 1, the robot moves faster due to smaller angle between the legs and torso leaning forward. Thus we can achieve both speed and stability by selecting Controller 1 when the ground is estimated to be close to level, while Controller 2 becomes the more stable choice as either uphill or downhill variations in upcoming terrain increase. A simple (non-optimal) switching algorithm is implemented based on this strategy and is shown in the Fig. 9 to show the feasibility and enhanced stability of this approach in using noisy, one-step sensor information about the average slope of upcoming terrain. To verify (15)
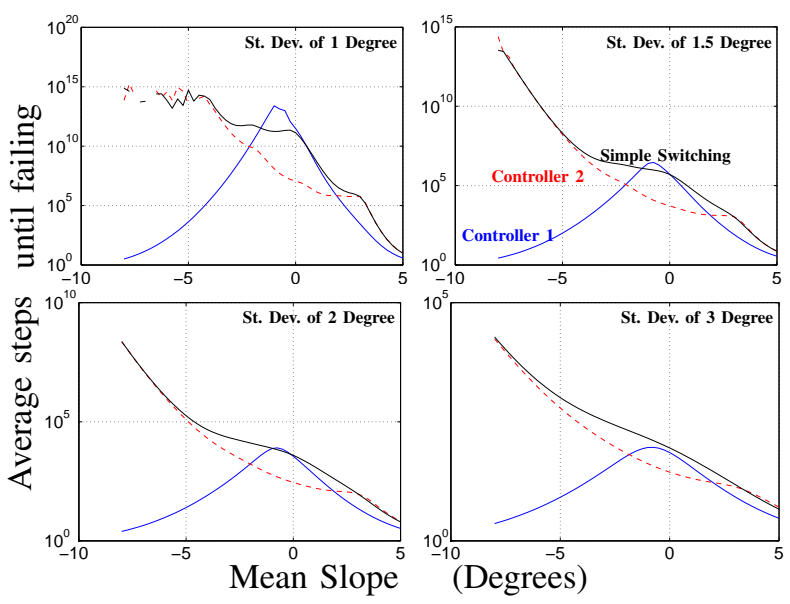

Fig. 9. Expected Mean Value of the Steps Before Failure

approximates adequately, we select four sample cases, i.e. controller, slope mean $\mu$ and deviation $\sigma$. We then use matrix $M$ to guess the number of steps before failure for randomly generated slopes using normal distribution. After a thousand 
failures were observed, we estimated the mean time to failure to obtain the results in Table V.

TABLE V

ESTIMATION OF NUMber of STEPS BEFORE FAILURE FOR KNOWN Controller, Mean Slope and Deviation

\begin{tabular}{|c|c|c|c|c|c|}
\hline Cont. & $\mu$ & $\sigma$ & Estimation by (15) & Estimation by (14) & Simulation \\
\hline 1 & -4 & 1.5 & 740.8 & 740.84 & 871.75 \\
\hline 1 & 0 & 3 & 71.45 & 72.041 & 87.32 \\
\hline 2 & 4 & 2 & 21.67 & 19.2928 & 18.88 \\
\hline 2 & 5 & 1 & 9.692 & 8.0525 & 9.693 \\
\hline
\end{tabular}

\section{CONCLUSION}

In this paper, we present a simple structure for controller which is later verified to exhibit finite time convergence. Thanks to the structure of the reference sets selected, we achieve a large ground clearance for each controller while maintaining stability. We use Poincaré methods to map the step-to-step transitions for states just before the impacts. We then show that switching between controllers yields gentle, flat surfaces in these Poincaré maps. Results show that the choice of controller for the previous step has limited , which indicates decoupling. We then created transition matrices of some particular examples of stochastic terrain, to see the big picture. We identified the safe regions in state space, in which a walker can be guaranteed to walk forever without falling, for particular bounds on terrain noise. We compared Monte Carlo trial estimate with estimated failure rates for a few example cases, toward verifying the eigenvalue analysis of [12] used to plot the expected mean value of the steps before failure. In light of these plots, we conclude that a simple control strategy can significantly increase the expected step until failure, given a noisy onestep estimate of next terrain slope. This work is motivated by the need for better control solutions for underactuated walkers to plan unsteady walking gaits on terrain for which upcoming information is available but corrupted with sensor noise. We conclude that by employing a set of discrete set of controllers, a bipedal robot is capable of moving it's impact state in the ten dimensional space, which can be mapped to real-life tasks and objectives, and we provide tools to analyze the resulting stability of such control policies.

\section{FUTURE WORK}

The authors currently plan to modify the 5-link biped model in the following ways. First, a lock-in mechanism such as the one outlined in [19] will be adopted for the knees. Secondly, an impulsive toe-off as described in [6] and [20] will be integrated. Thirdly, the impact event will be improved with springs and dampers at the expense of complexity. Then, we are planning to optimize the controller and to have a continuous reference set. At present, we have only anedotal evidence that such an approach is feasible; quantifying stability in this case remains an open challenge.
One particular goal is to create an adaptive biped that can adjust to given tasks and specifications while staying inside a bounded, stable region. One specific task we want to perform is to better control the swing tip height to avoid undesirable impacts. This can be done by changing $q_{4}^{\text {ref } 1}$ and the condition which determines $q_{4}^{\text {ref }}$. Examination of robustness to changes in model parameters is another topic we will be looking at. Our work here presents compelling evidence that applying our stochastic modeling techniques to high-dimensional robots is a practical goal, allowing one to quantify the trade-off between a wide range of goals (energetics, speed, foothold selection, etc.) and corresponding changes in the risk of failure.

\section{REFERENCES}

[1] M. Raibert, K. Blankespoor, G. Nelson, R. Playter, and the BigDog Team, "Bigdog, the rough-terrain quadruped robot," Proc. of the 17th World Congress, The Int. Fed. of Automatic Control (IFAC), 2008.

[2] H.-W. Park, K. Sreenath, A. Ramezani, and J. Grizzle, "Switching control design for accommodating large step-down disturbances in bipedal robot walking," in Proc. IEEE Int. Conf. on Robotics and Automation (ICRA), pp. 45 -50, 2012.

[3] J. C. P. A. Bhounsule and A. Ruina, "Design and control of Ranger: an energy-efficient, dynamic walking robot," in Proc. CLAWAR, pp. 441448, 2012.

[4] T. McGeer, "Passive dynamic walking," Internation Journal of Robotics Research, vol. 9, no. 2, pp. 62-82, 1990.

[5] K. Byl, Metastable Legged-Robot Locomotion. PhD thesis, MIT, 2008.

[6] K. Byl and R. Tedrake, "Approximate optimal control of the compass gait on rough terrain," in Proc. IEEE Int. Conf. on Robotics and Automation (ICRA), 2008.

[7] R. Tedrake, "LQR-Trees: Feedback motion planning on sparse randomized trees," in Proc. Robotics: Science and Systems (RSS), pp. 1-8, 2009.

[8] F. Iida and R. Tedrake, "Minimalistic control of a compass gait robot in rough terrain," in Proc. IEEE Int. Conf. on Robotics and Automation (ICRA), IEEE/RAS, 2009.

[9] R. D. M. Gregg, Geometric Control and Motion Planning for ThreeDimensional Bipedal Locomotion. PhD thesis, University of Illinois at Urbana-Champaign, 2010.

[10] I. R. Manchester, U. Mettin, F. Iida, and R. Tedrake, "Stable dynamic walking over uneven terrain," Int. J. of Robotics Research, vol. 30, March 2011.

[11] C. M. Hubicki, "Energy-economical heuristically based control of compass gait walking on stochastically varying terrain," Master's thesis, Bucknell Universitiy, 2011.

[12] K. Byl and R. Tedrake, "Metastable walking machines," Int. J. of Robotics Research, vol. 28, pp. 1040-1064, Aug 2009.

[13] M. Y. Chen and K. Byl, "Analysis and control techniques for the compass gait with a torso walking on stochastically rough terrain," in Proc. of American Control Conference (ACC), 2012.

[14] E. R. Westervelt, J. W. Grizzle, C. Chevallereau, J. H. Choi, and B. Morris, Feedback Control of Dynamic Bipedal Robot Locomotion. CRC Press, 2007.

[15] T. Nanayakkara, K. Byl, H. Liu, X. Song, and T. Villabona, "Dominant sources of variability in passive walking," in Proc. IEEE Int. Conf. on Robotics and Automation (ICRA), pp. 1003 -1010, 2012.

[16] S. Mohseni-Vahed and Y. Qin, "Effect of different terrain parameters on walking," in Advances in Reconfigurable Mechanisms and Robots I (J. S. Dai, M. Zoppi, and X. Kong, eds.), pp. 389-397, 2012.

[17] Y. Hurmuzlu and D. B. Marghitu, "Rigid body collisions of planar kinematic chains with multiple contact points," Int. J. of Robotics Research, pp. 82-92, Feb 1994.

[18] A. Sabanovic and K. Ohnishi, Motion Control Systems. John Wiley \& Sons, 2011.

[19] V. H. Chen, "Passive dynamic walking with knees: A point foot model," Master's thesis, Massachusetts Inst. of Tech., 2007.

[20] T. McGeer, "Stability and control of two-dimensional biped walking," Tech. Rep. 1, Simon Fraser Uni., Sep 1988. 